\title{
ARTICLE
}

\section{GEOCHEMISTRY AND GEOCHRONOLOGY OF THE PALEOZOIC SEDIMENTARY ROCKS IN THE SHAR KHUTUL AREA, CENTRAL MONGOLIA}

\author{
Erdenechimeg D. ${ }^{1 *}$, Oyunchimeg T. ${ }^{1}$, Otgonbaatar D. ${ }^{1}$, Jitka Míková2, Tomurchudur Ch. ${ }^{3}$ \\ Dagva-Ochir L. ${ }^{1}$ Enkhdalai B. ${ }^{1}$, Delgerzaya P. , Sharav D. ${ }^{1}$ and A. Zavřelová ${ }^{2}$ \\ ${ }^{I}$ Institute of Paleontology and Geology, Mongolian Academy of Sciences, Ulaanbaatar, Mongolia \\ ${ }^{2}$ Czech Geological Survey \\ ${ }^{3}$ Gurvantalst LLC, Ulaanbaatar, Mongolia
}

ARTICLE INFO: Received: 17 Oct, 2018; Accepted: 25 Dec, 2018

\begin{abstract}
The study area is located in the central part of Tsetserleg terrane in the southwestern margin of the Khangai-Khentey orogenic system. The paper presents new data on geochemistry and geochronology of sedimentary rocks from the Shar Khutul area, where the Tsetserleg terrane consists of Silurian-Devonian oceanic plate stratigraphic unit and Carboniferous shallow water sediment. The Upper Silurian to Middle Devonian Erdenetsogt Formation ( $\left.S_{3}-D_{2} e r\right)$, which is an oceanic plate stratigraphic unit, is mainly composed of siliceous siltstone, volcanites, tuffs, quartzite, and cherts. The shallow water sediments are divided into Upper Devonian to Lower Carboniferous Tsetserleg Formation $\left(D_{3}-C_{1} c c\right)$ and Lower-Middle Carboniferous Dzargalant Formation $\left(C_{1-2} d z\right)$. The Tsetserleg Formation $\left(D_{3}-C_{1} c c\right)$ consists of only sedimentary rocks such as bluish-grey sandstones and siltstones, and Lower-Middle Carboniferous Dzargalant Formation $\left(C_{1-2} d z\right)$ is principally composed of medium- to coarse-grained, brown-greenish grey sandstones with thin-layers of dark siltstones and gravelites.

The $\mathrm{SiO}_{2}$ content of the Shar Khutul area sandstones ranges from 63.85 to $67.95 \mathrm{wt} \%$ and the average content of $\mathrm{TiO}_{2}$ is $0.72 \mathrm{wt} . \%$ and $\mathrm{Al}_{2} \mathrm{O}_{3}$ content is $14.38 \mathrm{wt} \%$. The Chemical Index of Alteration (CIA) value ranges from 48.71 to 56.94 and the range of Index of compositional variations (ICV) is from 0.98 to 1.24. Moreover, the samples studied show that most of the sandstones are generally immature and were derived from weakly weathered source rocks.

The ratios of $E u / E u^{*}$ (0.83), La/Sc (3.81), La/Co (5.30), and $\mathrm{Cr} / \mathrm{Th}$ (13.81) indicate that the derivation of the Shar Khutul area sandstones from felsic rock sources and confirm the signatures of a felsic igneous provenance and suggest an active continental margin tectonic setting of the source area.

The clastic zircons from the medium grained sandstone (Erdenetsogt formation) yield ages between $2.5 \mathrm{Ga}$ and $236 \mathrm{Ma}$ and the detrital zircons exhibit four peak ages at 1.7-2.5 $\mathrm{Ga}(\mathrm{n}=13), 455-499 \mathrm{Ma}(\mathrm{n}=6)$, 337$382 \mathrm{Ma}(n=13)$ and $236-250 \mathrm{Ma}(\mathrm{n}=5)$.
\end{abstract}

Keywords: Shar Khutul area, sedimentary rocks, geochemistry, geochronology, provenance, tectonic setting; 


\section{INTRODUCTION}

The Devonian-Carboniferous Tsetserleg terrane takes place in the Khangai mountain ranges. The terrane is divided into the LowerMiddle Devonian chert-basalt-terrigenous (Erdenetsogt Formation), Middle-Upper Devonian flyshoid (Tsetserleg Formation) and Lower Carboniferous (Dzargalant Formation) [38, 12, 15, 39]. A type-section of the Lower-Middle Devonian Erdenetsogt Formation $\left(\mathrm{D}_{1-2} \mathrm{er}\right)$ was made by Tomorchudur et al. [38] at about $15 \mathrm{~km}$ northeast from the village of Erdenetsogt soum and divided into two members. Lower member consists of polymictic, oligomictic conglomerate, sandstone and siltstone. Upper member is mainly composed of reddish-grey coloured chert-quartzite, metabasalt, conglo-breccia, and bluish, green-grey coloured sandstone,

\section{Geological setting}

Previous studies inferred that the age of the Tsetserleg Formation belongs to the MiddleUpper Devonian, the Erdenetsogt Formation is Lower-Middle Devonian, and Dzargalant Formation is Lower Carboniferous in age. We have modified the age of those Formations based on the field observation, geochemical and geochronological data. According to our study, the Erdenetsogt Formation is Upper Silurian-Middle Devonian, the Tsetserleg Formation is Upper Devonian-Lower Carboniferous, and Dzargalant Formation is Lower-Middle Carboniferous, respectively [10]. The study area is dominated by Upper Devonian-Lower Carboniferous Tsetserleg Formation $\left(\mathrm{D}_{3}-\mathrm{C}_{1} \mathrm{cc}\right)$ which consists of bluish grey siliceous siltstone, sandstone, gravelite and rarely radiolarian siltstone (Figure 1).

The Tsetserleg Formation borders on the Upper Silurian-Middle Devonian Erdenetsogt Formation $\left(\mathrm{S}_{3}-\mathrm{D}_{2}\right.$ er), which is recognized as an oceanic plate stratigraphic (OPS) succession and exposed by tectonic wedge (Figure 2). The Erdenetsogt Formation is lithologically conglomerate and siltstone. The MiddleUpper Devonian Tsetserleg Formation $\left(\mathrm{D}_{2-3} \mathrm{cc}\right)$ consists of blue, bluish, grey, fine-medium grained sandstones with thin layers of dark, dark-grey siltstones. The Lower Carboniferous Dzargalant Formation $\left(\mathrm{C}_{1} \mathrm{dz}\right)$ is represented by brownish green, brownish grey, medium-course grained polymictic, oligomictic sandstones, gravelites and siltstones. Numerous studies, which aimed at determining the provenance, source weathering, and tectonic setting, were made by some researchers $[24,29,30,2]$ in the Khangai region.

This paper presents provenance, depositional period, weathering condition and tectonic setting of sedimentary units according to their geochemical analysis and geochronological data.

divided into three different sequences. The first sequence is characterized by whitishgrey coloured quartzite, greenschist facies metamorphosed basalt, which is classified into Upper Silurian-Lower Devonian. The second sequence is composed of greengreenish coloured basalt, red-reddish coloured chert, quartzite-jasperoid and tuff, which is classified into Lower Devonian. The third sequence consists of green coloured basalt, bluish-grey siltstone and tuff, which is classified into Lower-Middle Devonian. The Tsetserleg Formation is composed of greybluish coloured sandstone, siltstone and some layers of conglomerate and gravelite, which is conformably overlaid by Lower-Upper Carboniferous Dzargalant Formation $\left(\mathrm{C}_{1-2} \mathrm{dz}\right)$. Based on geochronological data of the detrital zircon and radiolarian fossils (palaeoscenidium sp., tetratormentum sp., albaillella sp.), the Tsetserleg Formation age is inferred to Upper Devonian-Lower Carboniferous period. The Dzargalant Formation consists of sandstone, siltstone and conglomerate, which is probably 
Lower-Upper Carboniferous. Its analogical unit is named by Dulaankhairkhan Formation $\left(\mathrm{C}_{1-2} \mathrm{dh}\right)$ in the southeastern part of the

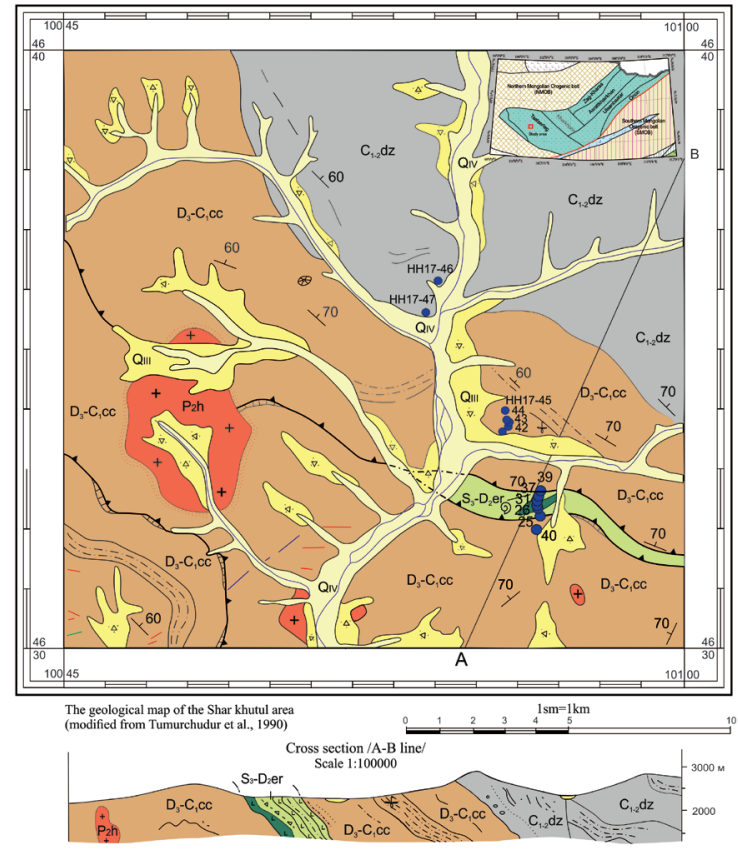

Tsetserleg terrane, which is dated as $348 \mathrm{Ma}$ on the detrital zircon [27].

Figure 1. The geological map of the Shar Khutul area modified after Tomorchudur et al. [38] and location of the study area [39].
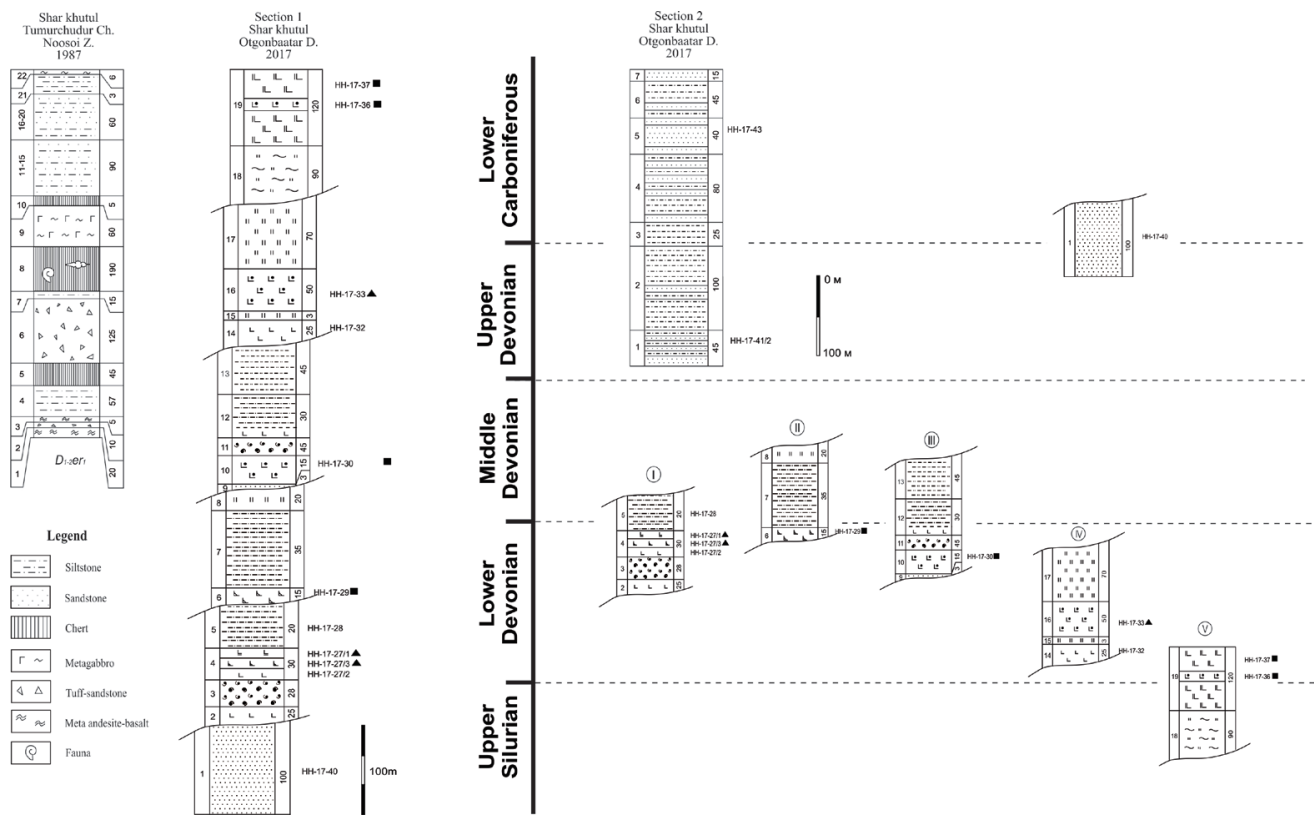

Figure 2. New schematic section of the Shar Khutul area 


\section{MATERIALS AND METHODS}

Nine thin sections of the sedimentary rocks from different units were studied using petrographic microscope and geochemical (major, trace and rare earth elements) analysis and one sample from the Tsetserleg Formation in the Shar Khutul area, Central Mongolia was dated by $\mathrm{U}-\mathrm{Pb}$ detrital zircon dating.

\section{Geochemical analysis}

The samples were analysed for major, trace and rare earth elements (REE) at SGS Mongolia LLC, Ulaanbaatar, Mongolia. The major elements ( $\mathrm{Si}, \mathrm{Ti}, \mathrm{Al}, \mathrm{Fe}, \mathrm{Mn}, \mathrm{Mg}, \mathrm{Ca}, \mathrm{Na}$, $\mathrm{K}$ and $\mathrm{P}$ ) were analysed by $\mathrm{X}$-ray fluorescence spectrometer (XRF), and trace and rare earth elements (54 elements) by inductively coupled plasma mass spectrometry (ICP-MS).

\section{$U$-Pb zircon dating}

The samples were first crushed to 80-100 mesh, and the low-density materials were then removed using conventional heavy-

\section{RESULTS AND DISCUSSION}

\section{Petrography}

The petrographic analysis (Figure 3) shows that sandstones of Shar Khutul area are mainly characterized by medium to coarse grained particle. The sandstones are mostly poorly sorted with angular to subrounded grains. The main mineralogical constituents are quartz, feldspars, and lithic fragments (55-60\%). Quartz is the most predominant constituent mineral and is present as monocrystalline (Qm) and polycrystalline (Qp). Two types of feldspars have been identified, which liquid techniques. High-purity zircon samples were then separated from remaining heavy minerals using magnetic separation techniques at the Laboratory of Petrography, Institute of Paleontology and Geology, Mongolian Academy of Sciences, Ulaanbaatar, Mongolia. High-quality non-fractured zircon grains were inlaid in epoxy, polished down to half their original thickness and washed in an acid bath before analysis. The cathodoluminescence (CL) images were collected using field emission scanning electron microscope FEGSEM Tescan Mira3 GMU at the Center of Lithospheric Research of the Czech Geological Survey. Based on analysis of the CL images, distinct location within the zircons were selected as the best point for laser ablation inductively coupled plasma mass spectrometer (LA-ICP-MS). An Agilent 7900x Quad ICPMS equipped Analyte Excite 193nm excimer laser was used to measure the U-Pb isotope ages of the zircons.

include albite (plagioclase), orthoclase and microcline ( $\mathrm{k}$-feldspar). $\mathrm{K}$-feldspars is more abundant than plagioclase. Lithic fragments are predominantly composed of granite, mudstones, schist and andesite. Cement has a percentage range of 40 to 45 , and include very fine grained quartz, feldspars, mudstones, biotite, chlorite, carbonate, and iron oxide. The accessory minerals are composed of mainly non-opaque minerals (zircon, apatite, rutile, and minor tourmaline). 


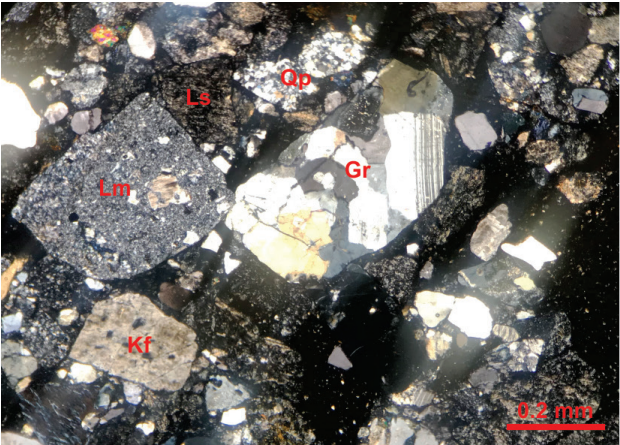

Figure 3. Photomicrographs of sandstones from the Shar Khutul area: a) HH-17-47 b) HH-17-50 (monocrystalline $(\mathrm{Qm})$ and polycrystalline $(Q p)$ quartz, plagioclase (Pl), K-feldsphar (Kf), metamorphic lithic fragment $(\mathrm{Lm})$ and a siltstone fragment $(\mathrm{Ls})$ ).

\section{Geochemical composition}

Table 1. Major and trace element analyses of the sandstones from the Shar Khutul area

\begin{tabular}{|c|c|c|c|c|c|c|c|c|c|}
\hline \multirow{3}{*}{ 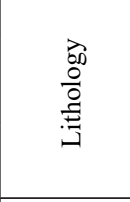 } & \multicolumn{5}{|c|}{ Tsetserleg Formation $\left(\mathrm{D}_{3}-\mathrm{C}_{1} \mathrm{cc}\right)$} & \multicolumn{4}{|c|}{ Dzargalant Formation $\left(\mathrm{C}_{1-2} \mathrm{dz}\right)$} \\
\hline & $\begin{array}{c}\mathrm{HH}-17- \\
40\end{array}$ & $\begin{array}{c}\mathrm{HH}-17- \\
41 / 2\end{array}$ & $\begin{array}{c}\mathrm{HH}-17- \\
43\end{array}$ & $\begin{array}{c}\mathrm{HH}-17- \\
44\end{array}$ & $\begin{array}{c}\mathrm{HH}-17- \\
45\end{array}$ & $\begin{array}{c}\mathrm{HH}-17- \\
46\end{array}$ & $\begin{array}{c}\mathrm{HH}-17- \\
47\end{array}$ & $\begin{array}{c}\mathrm{HH}-17- \\
48\end{array}$ & $\begin{array}{c}\mathrm{HH}-17- \\
50\end{array}$ \\
\hline & $\begin{array}{l}\text { Sand- } \\
\text { stone }\end{array}$ & $\begin{array}{l}\text { Sand- } \\
\text { stone }\end{array}$ & $\begin{array}{l}\text { Sand- } \\
\text { stone }\end{array}$ & $\begin{array}{l}\text { Sand- } \\
\text { stone }\end{array}$ & $\begin{array}{l}\text { Sand- } \\
\text { stone }\end{array}$ & $\begin{array}{l}\text { Sand- } \\
\text { stone }\end{array}$ & $\begin{array}{l}\text { Sand- } \\
\text { stone }\end{array}$ & $\begin{array}{l}\text { Sand- } \\
\text { stone }\end{array}$ & $\begin{array}{l}\text { Sand- } \\
\text { stone }\end{array}$ \\
\hline \multicolumn{10}{|c|}{ Major elements (wt.\%) } \\
\hline $\mathrm{SiO}_{2}$ & 66.86 & 65.38 & 66.04 & 63.85 & 66.20 & 66.58 & 65.37 & 67.95 & 64.40 \\
\hline $\mathrm{TiO}_{2}$ & 0.71 & 0.78 & 0.72 & 0.79 & 0.78 & 0.67 & 0.75 & 0.58 & 0.71 \\
\hline $\mathrm{Al}_{2} \mathrm{O}_{3}$ & 15.93 & 14.22 & 14.29 & 14.63 & 13.93 & 14.43 & 14.41 & 13.66 & 13.96 \\
\hline $\mathrm{Fe}_{2} \mathrm{O}_{3}$ & 4.65 & 4.64 & 4.51 & 5.30 & 4.45 & 4.17 & 4.72 & 4.16 & 5.46 \\
\hline $\mathrm{MnO}$ & 0.09 & 0.07 & 0.07 & 0.07 & 0.07 & 0.07 & 0.07 & 0.07 & 0.09 \\
\hline $\mathrm{MgO}$ & 1.39 & 1.59 & 1.53 & 2.04 & 1.36 & 1.48 & 1.56 & 1.41 & 1.90 \\
\hline $\mathrm{CaO}$ & 1.41 & 1.86 & 2.03 & 2.20 & 2.40 & 1.78 & 2.28 & 2.03 & 2.46 \\
\hline $\mathrm{Na}_{2} \mathrm{O}$ & 3.55 & 4.04 & 4.41 & 3.60 & 4.67 & 4.23 & 4.03 & 3.60 & 3.87 \\
\hline $\mathrm{K}_{2} \mathrm{O}$ & 3.85 & 2.95 & 2.81 & 3.03 & 2.71 & 3.02 & 3.15 & 2.99 & 2.80 \\
\hline $\mathrm{P}_{2} \mathrm{O}_{5}$ & 0.22 & 0.17 & 0.17 & 0.22 & 0.13 & 0.18 & 0.20 & 0.12 & 0.16 \\
\hline LOI & 1.66 & 2.85 & 2.08 & 2.84 & 1.89 & 2.02 & 1.99 & 2.40 & 2.69 \\
\hline Total & 100.32 & 98.55 & 98.66 & 98.57 & 98.59 & 98.63 & 98.53 & 98.97 & 98.50 \\
\hline $\begin{array}{l}\mathrm{SiO}_{2} / \\
\mathrm{Al}_{2} \mathrm{O}_{3}\end{array}$ & 4.20 & 4.60 & 4.62 & 4.36 & 4.75 & 4.61 & 4.54 & 4.97 & 4.61 \\
\hline $\mathrm{K}_{2} \mathrm{O} / \mathrm{Al}_{2} \mathrm{O}_{3}$ & 0.24 & 0.21 & 0.20 & 0.21 & 0.19 & 0.21 & 0.22 & 0.22 & 0.20 \\
\hline $\mathrm{K}_{2} \mathrm{O} / \mathrm{Na}_{2} \mathrm{O}$ & 1.08 & 0.73 & 0.64 & 0.84 & 0.58 & 0.71 & 0.78 & 0.83 & 0.72 \\
\hline $\mathrm{Na}_{2} \mathrm{O} / \mathrm{K}_{2} \mathrm{O}$ & 0.92 & 1.37 & 1.57 & 1.19 & 1.72 & 1.40 & 1.28 & 1.20 & 1.38 \\
\hline $\begin{array}{c}\mathrm{Al}_{2} \mathrm{O}_{3} / \\
\mathrm{TiO}_{2} \\
\end{array}$ & 22.44 & 18.23 & 19.85 & 18.52 & 17.86 & 21.54 & 19.21 & 23.55 & 19.66 \\
\hline
\end{tabular}


Vol. 58 No 04 (228) 2018

\begin{tabular}{|c|c|c|c|c|c|c|c|c|c|}
\hline F1 & -1.16 & -0.75 & -0.20 & -0.81 & 0.17 & -0.73 & -0.53 & -1.08 & -0.14 \\
\hline F2 & 3.30 & 2.91 & 3.34 & 1.82 & 3.96 & 3.39 & 3.31 & 2.49 & 2.05 \\
\hline CIA & 56.94 & 52.60 & 51.27 & 53.58 & 48.71 & 52.54 & 51.25 & 52.09 & 50.86 \\
\hline ICV & 0.98 & 1.12 & 1.12 & 1.16 & 1.18 & 1.07 & 1.15 & 1.09 & 1.24 \\
\hline
\end{tabular}

Trace elements (ppm)

\begin{tabular}{|c|c|c|c|c|c|c|c|c|c|}
\hline $\mathrm{Rb}$ & 106.00 & 82.50 & 71.20 & 89.20 & 60.50 & 77.80 & 77.20 & 84.10 & 74.50 \\
\hline $\mathrm{Ba}$ & 967.00 & 1048.0 & 857.00 & 937.0 & 693.00 & 997.0 & 754.00 & 782.00 & 967.00 \\
\hline $\mathrm{Sr}$ & 306.00 & 373.00 & 300.00 & 344.0 & 335.00 & 367.0 & 330.00 & 319.00 & 345.00 \\
\hline $\mathrm{Th}$ & 8.80 & 7.80 & 6.60 & 7.40 & 6.10 & 5.80 & 7.40 & 8.00 & 6.70 \\
\hline $\mathrm{U}$ & 2.03 & 2.13 & 1.79 & 2.02 & 1.48 & 1.48 & 2.09 & 2.34 & 1.90 \\
\hline $\mathrm{Zr}$ & 250.00 & 271.00 & 212.00 & 211.0 & 284.00 & 205.0 & 217.00 & 288.00 & 293.00 \\
\hline Hf & 7.00 & 7.00 & 6.00 & 6.00 & 6.00 & 5.00 & 5.00 & 6.00 & 6.00 \\
\hline $\mathrm{Y}$ & 27.70 & 24.90 & 24.50 & 26.90 & 20.40 & 22.80 & 25.40 & 25.20 & 23.30 \\
\hline $\mathrm{Nb}$ & 10.00 & 9.00 & 8.00 & 9.00 & 7.00 & 7.00 & 8.00 & 8.00 & 7.00 \\
\hline $\mathrm{Sc}$ & 10.00 & 9.00 & 10.00 & 12.00 & 8.00 & 11.00 & 9.00 & 8.00 & 10.00 \\
\hline $\mathrm{V}$ & 59.00 & 69.00 & 70.00 & 87.00 & 69.00 & 78.00 & 60.00 & 50.00 & 77.00 \\
\hline $\mathrm{Cr}$ & 46.00 & 98.00 & 82.00 & 112.0 & 102.00 & 110.0 & 96.00 & 68.00 & 146.00 \\
\hline $\mathrm{Co}$ & 4.30 & 6.90 & 6.80 & 7.80 & 8.10 & 5.70 & 7.90 & 7.10 & 10.80 \\
\hline $\mathrm{Cu}$ & 10.00 & 11.00 & 10.00 & 19.00 & 10.00 & 15.00 & 10.00 & 17.00 & 10.00 \\
\hline $\mathrm{Ni}$ & 5.00 & 21.00 & 12.00 & 59.00 & 7.00 & 10.00 & 29.00 & 9.00 & 50.00 \\
\hline $\mathrm{Zn}$ & 55.00 & 64.00 & 70.00 & 96.00 & 57.00 & 64.00 & 75.00 & 76.00 & 94.00 \\
\hline $\mathrm{Th} / \mathrm{U}$ & 4.33 & 3.66 & 3.69 & 3.66 & 4.12 & 3.92 & 3.54 & 3.42 & 3.53 \\
\hline $\mathrm{Th} / \mathrm{Sc}$ & 0.88 & 0.87 & 0.66 & 0.62 & 0.76 & 0.53 & 0.82 & 1.00 & 0.67 \\
\hline $\mathrm{Zr} / \mathrm{Sc}$ & 25.00 & 30.11 & 21.20 & 17.58 & 35.50 & 18.64 & 24.11 & 36.00 & 29.30 \\
\hline $\mathrm{Cr} / \mathrm{Th}$ & 5.23 & 12.56 & 12.42 & 15.14 & 16.72 & 18.97 & 12.97 & 8.50 & 21.79 \\
\hline
\end{tabular}

CIA $(\%)=\left[\mathrm{Al}_{2} \mathrm{O}_{3} /\left(\mathrm{Al}_{2} \mathrm{O}_{3}+\mathrm{CaO} *+\mathrm{Na}_{2} \mathrm{O}+\mathrm{K}_{2} \mathrm{O}\right)\right] \times 100$

$\mathrm{ICV}=\left(\mathrm{FeO}_{3}+\mathrm{K}_{2} \mathrm{O}+\mathrm{Na}_{2} \mathrm{O}+\mathrm{CaO}+\mathrm{MgO}+\mathrm{TiO}_{2}\right) / \mathrm{Al}_{2} \mathrm{O}_{3} \cdot \mathrm{CaO} *=\mathrm{CaO}-\left[\mathrm{P}_{2} \mathrm{O}_{5} \times(10 / 3)\right]$;

$\mathrm{F} 1=\left(-1.773 \mathrm{xTiO}_{2}\right)+\left(0.607 \mathrm{xAl}_{2} \mathrm{O}_{3}\right)+\left(0760 \mathrm{xFe}_{2} \mathrm{O}_{3}\right)+(-1.500 \mathrm{xMgO})+(0.616 \mathrm{xCaO})+\left(0.509 \mathrm{xNa}_{2} \mathrm{O}\right)+$

$+\left(-1.224 \mathrm{xK}_{2} \mathrm{O}\right)+(-9.09)$

$\mathrm{F} 2=\left(0.445 \mathrm{xTiO}_{2}\right)+\left(0.070 \mathrm{xAl}_{2} \mathrm{O}_{3}\right)+\left(-0.250 \mathrm{xFe}_{2} \mathrm{O}_{3}\right)+(1.142 \mathrm{xMgO})+(0.438 \mathrm{xCaO})+\left(1.475 \mathrm{xNa}_{2} \mathrm{O}\right)+$

$+\left(1.426 \mathrm{xK}_{2} \mathrm{O}\right)+(-6.861)$

\section{Major elements}

The $\mathrm{SiO}_{2}$ content of the Tsetserleg Formation sandstones of the Shar Khutul area ranges from 63.85 to $66.86 \mathrm{wt} . \%$ with an average of 65.67 wt. $\%, \mathrm{~K}_{2} \mathrm{O} / \mathrm{Na}_{2} \mathrm{O}$ ratio is 0.77, and the Dzargalant Formation sandstones have $\mathrm{SiO}_{2}$ content ranging from 64.40 to 67.95 wt. $\%$ (av. $=66.08$ wt. $\%$ ), $\mathrm{K}_{2} \mathrm{O} / \mathrm{Na}_{2} \mathrm{O}$ ratio is 0.76 (Table 1). The average values of $\mathrm{Al}_{2} \mathrm{O}_{3}$ range between 14.11 and 14.60. Variations in the major geochemistry elements of the Shar Khutul area sandstones are shown on Harker diagrams (Figure 4). $\mathrm{Al}_{2} \mathrm{O}_{3}$ against major oxides were plotted on variation diagrams, $\mathrm{K}_{2} \mathrm{O}, \mathrm{P}_{2} \mathrm{O}_{5}, \mathrm{TiO}_{2}, \mathrm{Fe}_{2} \mathrm{O}_{3}, \mathrm{MgO}$ and $\mathrm{MnO}$ show positive correlation with linear trend. $\mathrm{CaO}$, $\mathrm{Na}_{2} \mathrm{O}$ and $\mathrm{SiO}_{2}$ negatively correlated with $\mathrm{Al}_{2} \mathrm{O}_{3}$. 

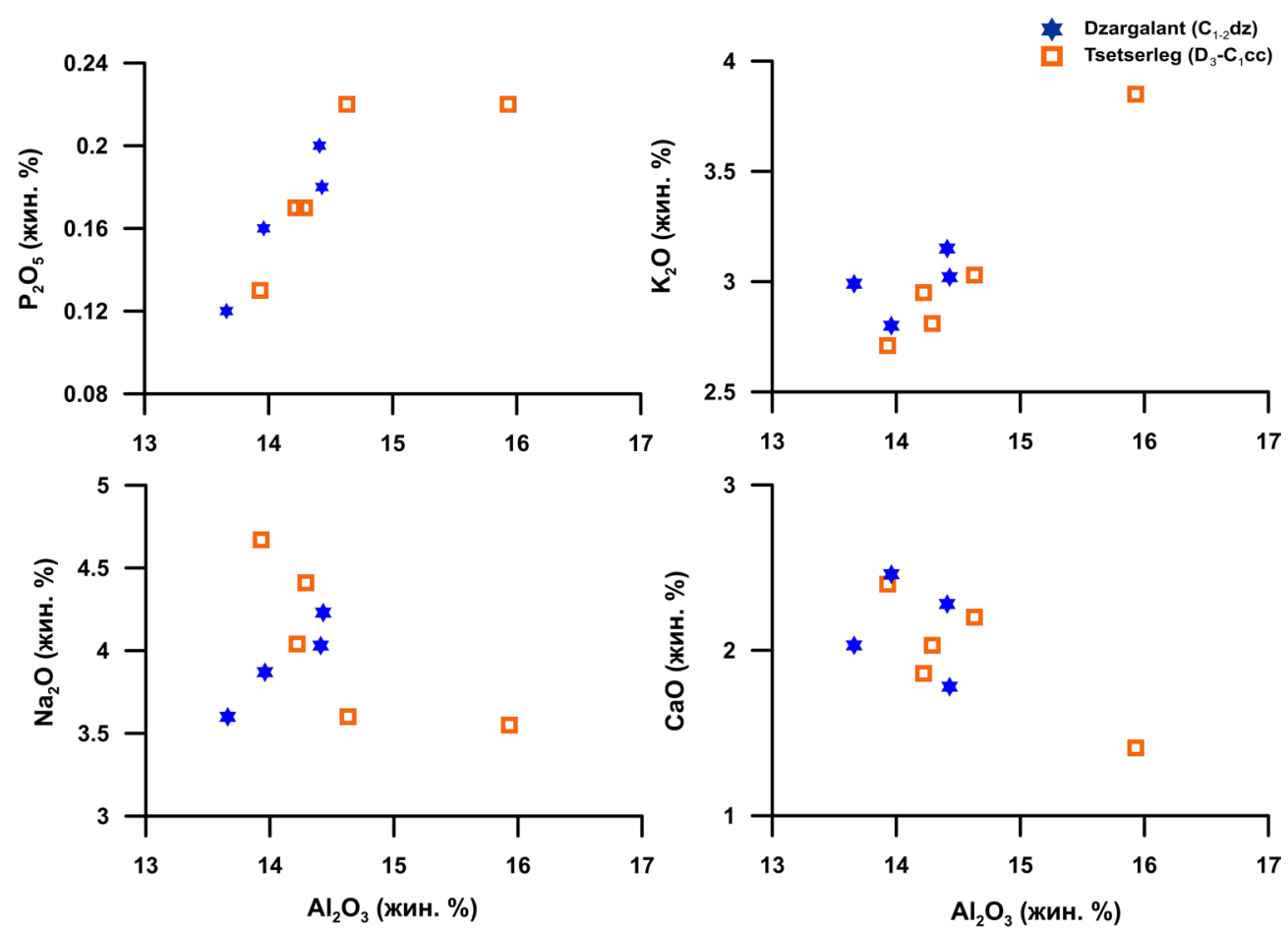

Figure 4. Harker variation diagrams for the Shar Khutul area sandstones

In addition, the $\mathrm{K}_{2} \mathrm{O}$ and $\mathrm{Na}_{2} \mathrm{O}$ contents of sandstone samples and their ratios $\left(\mathrm{Na}_{2} \mathrm{O} /\right.$ $\mathrm{K}_{2} \mathrm{O}>1$ ) are consistent with the petrographic observations, in which plagioclase dominates over $\mathrm{K}$-feldspar, and the $\mathrm{Na}_{2} \mathrm{O} / \mathrm{K}_{2} \mathrm{O}$ ratio is more than 1 , therefore, the sandstones $\mathrm{Na}_{2} \mathrm{O}$ / $\mathrm{K}_{2} \mathrm{O}$ ratio represent immature sediments near to the source (Table 1) [16]. Thus, the sediments $\mathrm{K}_{2} \mathrm{O} / \mathrm{Al}_{2} \mathrm{O}_{3}$ ratio can be used as an indicator of ancient sediments original composition. The $\mathrm{K}_{2} \mathrm{O} / \mathrm{Al}_{2} \mathrm{O}_{3}$ ratios for clay minerals and feldspars are different ( 0.0 to 0.3 , 0.3 to 0.9 , respectively) according to Cox [6]. In this present study, the average $\mathrm{K}_{2} \mathrm{O} / \mathrm{Al}_{2} \mathrm{O}_{3}$ ratio of the sandstone is 0.21 , which is their original composition may have been enriched by clay minerals.

The sandstones of the Shar Khutul area with their chemical composition are greywacke affinity (Figure 5).

The composition of major element in sandstones has also been used to determine sedimentary provenance by the application of discriminant function analysis [34].In this discrimination diagram, sandstones of both the formations are plotted in the felsic igneous provenance (Figure 6). 

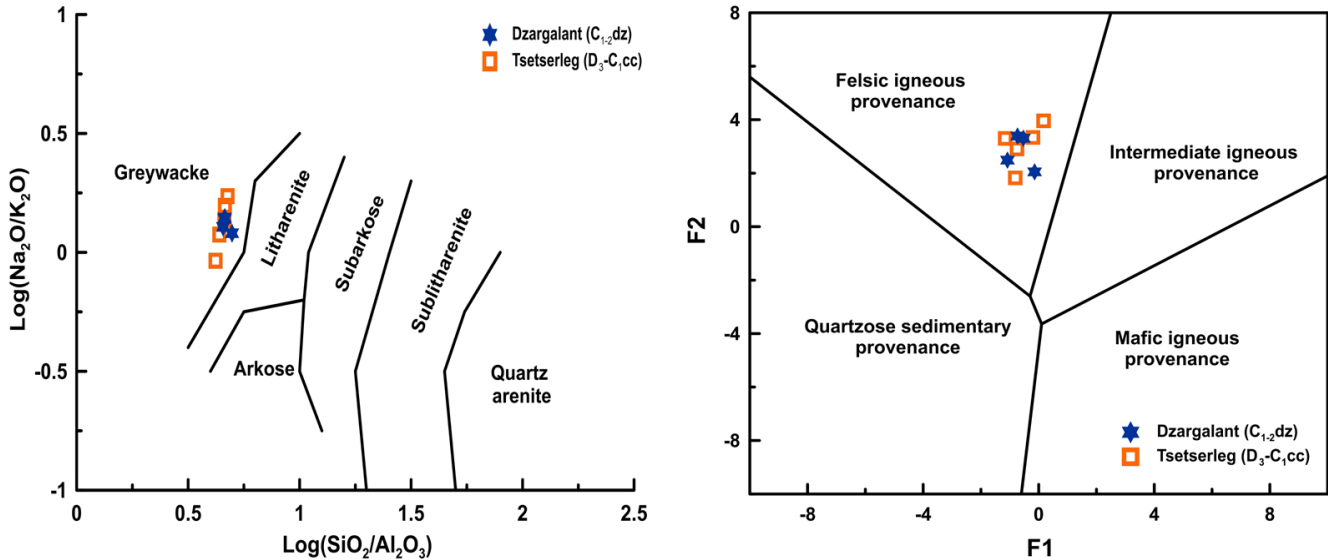

Figure 5. Geochemical classification of the sandstones [28]

Figure 6. Discriminant function diagram using major elements for the provenance signature of the sedimentary rocks [34]

\section{Trace elements}

Trace elements, including $\mathrm{Cr}, \mathrm{Ni}, \mathrm{V}, \mathrm{Co}$, $\mathrm{Y}, \mathrm{Sc}, \mathrm{Zr}$, Hf and $\mathrm{Th}$, reside in minerals that are resistant to secondary processes and are commonly used to constrain the composition of the source $[37,21,40]$. The relative abundance of $\mathrm{Co}$ and $\mathrm{Cr}$ (indicative of mafic source), $\mathrm{La}$ and Th (indicative of felsic source) and ratio such as $\mathrm{Co} / \mathrm{Th}$ and $\mathrm{La} / \mathrm{Sc}$ are generally used to examine the geochemical nature of the source rocks [18, 9, 40, 41]. The sandstones from the Shar Khutul area have $\mathrm{Co} / \mathrm{Th}$ and $\mathrm{La} /$ $\mathrm{Sc}$ ratios generally similar to $\mathrm{UCC}$, indicating

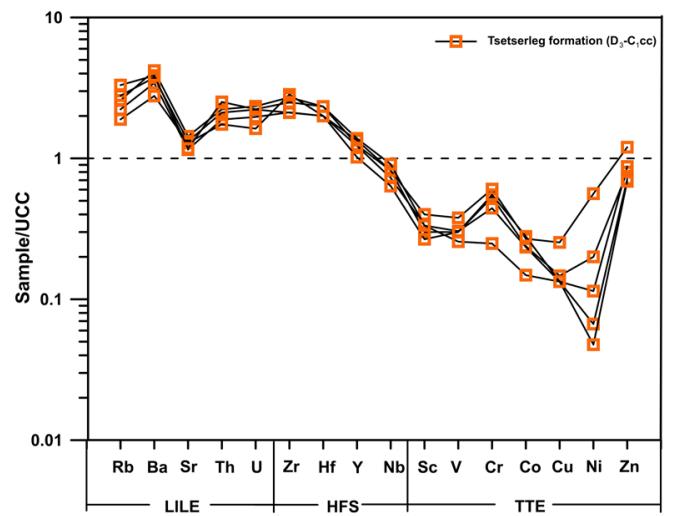

dominantly felsic sources. Furthermore, depletion of compatible elements $(\mathrm{Cr}, \mathrm{V}, \mathrm{Ni}$ and $\mathrm{Sc}$ ) and slight enrichment of HFSE (Zr and Hf) relative to the UCC, are consistent with such felsic source.

The upper continental crust-normalized trace element diagram shows high content in large ion lithophile elements (LILE) and high field strength elements (HFSE), lower content in transition trace elements (TTE), therefore, we believe that their origin is derived from felsic source rocks (Figure 7).

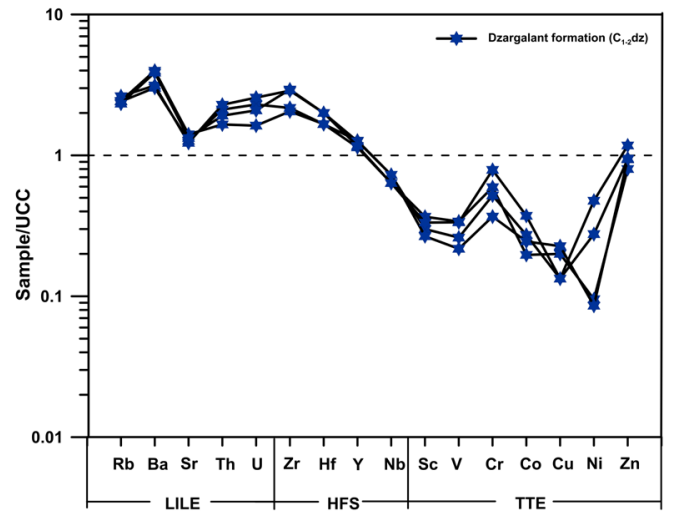

Figure 7. Upper continental crust normalized trace elements pattern. UCC normalized data are from Taylor and McLennan [37] 


\section{Rare earth elements}

The REE patterns were used to infer sources of sedimentary rocks. The $\mathrm{Eu} / \mathrm{Eu}^{*}$ (0.83), $\mathrm{La} / \mathrm{Sc}$ (3.81), $\mathrm{La} / \mathrm{Co}$ (5.30) ratios indicate derivation of the Shar Khutul area sandstones from felsic rock sources.

The Tsetserleg Formation sandstones have
$(\mathrm{La} / \mathrm{Yb}) \mathrm{N}$ ratios ranging from 7.81 to 11.18 (av. $=9.48)$ and the Dzargalant Formation sandstones range from 9.32 to 10.36 $(\mathrm{av} .=9.96)$. The rare earth element contents of the sandstones from the Shar Khutul area are presented in Table 2.

Table 2. Rare earth element concentrations of sandstones from the Shar Khutul area

\begin{tabular}{|c|c|c|c|c|c|c|c|c|c|}
\hline \multirow{3}{*}{ Lithology } & \multicolumn{5}{|c|}{ Tsetserleg Formation $\left(\mathrm{D}_{3}-\mathrm{C}_{1} \mathrm{cc}\right)$} & \multicolumn{4}{|c|}{ Dzargalant Formation $\left(\mathrm{C}_{1-2} \mathrm{dz}\right)$} \\
\hline & $\begin{array}{c}\mathrm{HH}-17- \\
40\end{array}$ & $\begin{array}{c}\mathrm{HH}-17- \\
41 / 2\end{array}$ & $\begin{array}{c}\mathrm{HH}-17- \\
43\end{array}$ & $\begin{array}{c}\mathrm{HH}-17- \\
44\end{array}$ & $\begin{array}{c}\mathrm{HH}-17- \\
45\end{array}$ & $\begin{array}{c}\mathrm{HH}-17- \\
46\end{array}$ & $\begin{array}{c}\mathrm{HH}-17- \\
47\end{array}$ & $\begin{array}{c}\mathrm{HH}-17- \\
48\end{array}$ & $\begin{array}{c}\mathrm{HH}-17- \\
50\end{array}$ \\
\hline & $\begin{array}{l}\text { Sand } \\
\text { stone }\end{array}$ & $\begin{array}{l}\text { Sand } \\
\text { stone }\end{array}$ & $\begin{array}{l}\text { Sand } \\
\text { stone }\end{array}$ & $\begin{array}{l}\text { Sand } \\
\text { stone }\end{array}$ & $\begin{array}{l}\text { Sand } \\
\text { stone }\end{array}$ & $\begin{array}{l}\text { Sand } \\
\text { stone }\end{array}$ & $\begin{array}{l}\text { Sand } \\
\text { stone }\end{array}$ & $\begin{array}{l}\text { Sand } \\
\text { stone }\end{array}$ & $\begin{array}{l}\text { Sand } \\
\text { stone }\end{array}$ \\
\hline \multicolumn{10}{|c|}{ Rare earth elements (ppm) } \\
\hline La & 37.00 & 37.10 & 35.30 & 35.80 & 34.30 & 36.60 & 39.00 & 35.10 & 36.50 \\
\hline $\mathrm{Ce}$ & 75.20 & 70.30 & 65.40 & 71.20 & 63.40 & 66.40 & 72.90 & 68.10 & 65.40 \\
\hline $\mathrm{Pr}$ & 7.93 & 7.80 & 7.28 & 7.86 & 7.04 & 7.15 & 7.86 & 7.48 & 7.36 \\
\hline $\mathrm{Nd}$ & 31.50 & 29.80 & 29.30 & 31.30 & 27.20 & 28.00 & 30.90 & 29.40 & 29.50 \\
\hline Sm & 7.20 & 6.30 & 6.20 & 6.90 & 5.20 & 5.70 & 6.30 & 6.60 & 6.10 \\
\hline $\mathrm{Eu}$ & 1.55 & 1.55 & 1.54 & 1.46 & 1.46 & 1.54 & 1.63 & 1.36 & 1.54 \\
\hline Gd & 5.63 & 5.22 & 4.99 & 5.51 & 4.25 & 4.74 & 5.23 & 5.20 & 4.66 \\
\hline $\mathrm{Tb}$ & 0.80 & 0.74 & 0.73 & 0.82 & 0.61 & 0.67 & 0.79 & 0.76 & 0.67 \\
\hline Dy & 4.96 & 4.48 & 4.35 & 4.89 & 3.77 & 4.21 & 4.71 & 4.45 & 4.24 \\
\hline Ho & 1.04 & 0.89 & 0.89 & 0.95 & 0.74 & 0.85 & 0.88 & 0.89 & 0.85 \\
\hline $\mathrm{Er}$ & 3.13 & 2.78 & 2.53 & 2.76 & 2.10 & 2.53 & 2.83 & 2.62 & 2.55 \\
\hline $\mathrm{Tm}$ & 0.47 & 0.41 & 0.37 & 0.42 & 0.33 & 0.36 & 0.40 & 0.39 & 0.38 \\
\hline $\mathrm{Yb}$ & 3.40 & 2.70 & 2.70 & 2.80 & 2.20 & 2.60 & 2.70 & 2.70 & 2.60 \\
\hline Lu & 0.57 & 0.38 & 0.37 & 0.40 & 0.32 & 0.40 & 0.41 & 0.44 & 0.34 \\
\hline$\sum$ REE & 180.38 & 170.45 & 161.95 & 173.1 & 152.92 & 161.75 & 176.54 & 165.49 & 162.69 \\
\hline $\begin{array}{l}\text { LREE/ } \\
\text { HREE }\end{array}$ & 11.55 & 12.77 & 12.56 & 12.27 & 14.19 & 12.92 & 12.88 & 12.51 & 12.99 \\
\hline $\mathrm{Eu} / \mathrm{Eu}^{*}$ & 0.74 & 0.83 & 0.85 & 0.72 & 0.95 & 0.91 & 0.87 & 0.71 & 0.88 \\
\hline $\mathrm{Ce} / \mathrm{Ce}^{*}$ & 1.08 & 1.01 & 1.00 & 1.04 & 1.00 & 1.01 & 1.02 & 1.03 & 0.98 \\
\hline $\mathrm{La} / \mathrm{Sc}$ & 3.70 & 4.12 & 3.53 & 2.98 & 4.29 & 3.33 & 4.33 & 4.39 & 3.65 \\
\hline $\mathrm{La} / \mathrm{Co}$ & 8.60 & 5.38 & 5.19 & 4.59 & 4.23 & 6.42 & 4.94 & 4.94 & 3.38 \\
\hline $\mathrm{La} / \mathrm{Th}$ & 4.20 & 4.76 & 5.35 & 4.84 & 5.62 & 6.31 & 5.27 & 4.39 & 5.45 \\
\hline$(\mathrm{La} / \mathrm{Sm})_{\mathrm{N}}$ & 3.32 & 3.80 & 3.68 & 3.35 & 4.26 & 4.15 & 4.00 & 3.43 & 3.86 \\
\hline$(\mathrm{La} / \mathrm{Yb})_{\mathrm{N}}$ & 7.81 & 9.86 & 9.38 & 9.17 & 11.18 & 10.10 & 10.36 & 9.32 & 10.07 \\
\hline$(G d / Y b)_{N}$ & 1.37 & 1.60 & 1.53 & 1.63 & 1.60 & 1.51 & 1.60 & 1.59 & 1.48 \\
\hline
\end{tabular}


The basic rocks generally have low LREE/ HREE ratios and no Eu anomalies, whereas, felsic rocks usually contain high LREE/HREE ratios and negative Eu anomalies [7, 8]. In this study, the sandstones are characterized by high
LREE/HREE ratios $(11.55-14.19$, av. $=12.74)$ and negative $\mathrm{Eu}$ anomalies $\left(\mathrm{Eu} / \mathrm{Eu}^{*}=0.71\right.$ 0.95 , av. $=0.83$ ), therefore, we consider that their origin derived from felsic source rocks, which is similar to UCC (Figure 8).
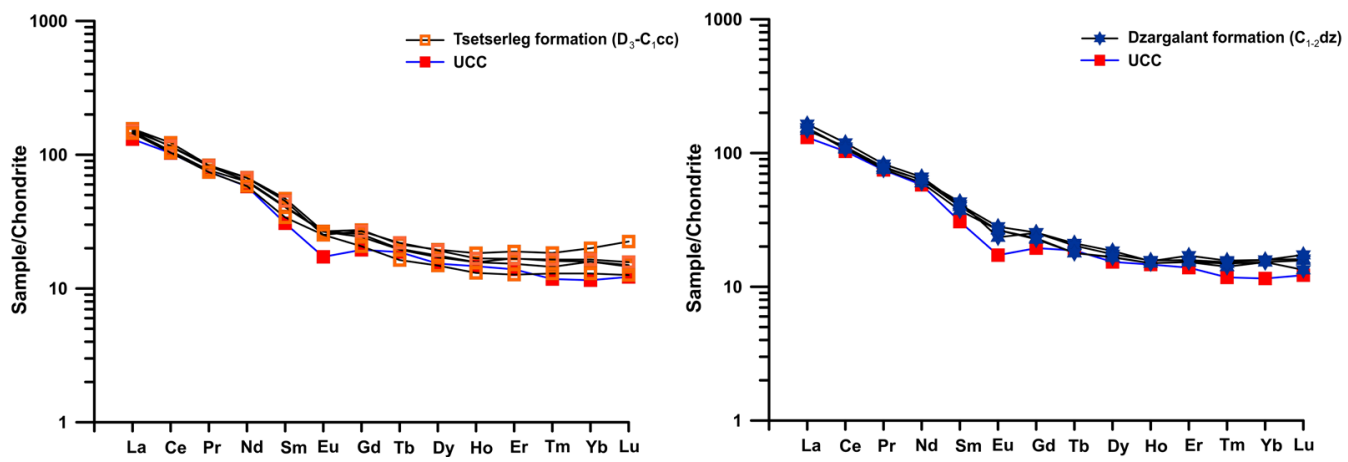

Figure 8. Chondrite normalized rare earth elements pattern. Chondrite-normalized values from Sun and McDonough [36]

\section{U-Pb zircon dating}

The sample was collected from the Erdenetsogt Formation of the Shar Khutul area, and is a bluish grey, medium grained sandstone (HH-17-40- N46031'58,6'; E10056”34.6'). Cathodoluminescence (CL) images clearly show that most of the analysed zircons euhedral to subhedral, with a few subrounded grains (Figure 9a).
In total, 52 single detrital zircon grains were analyzed from this sample, and 37 grains are selected for the final interpretation (Table 3). The sample yielded ages between $2.5 \mathrm{Ga}$ and $236 \mathrm{Ma}$, and the detrital zircons exhibit four peak ages at 1.7-2.5 $\mathrm{Ga}(\mathrm{n}=13), 455-499$ Ma $(n=6), 337-382 \mathrm{Ma}(\mathrm{n}=13)$ and 236-250 $\mathrm{Ma}(\mathrm{n}=5)$. (Figure 9b,c).

Table 3. Sample HH-17-40 U-Pb detrital zircon LA-ICP-MS analysis results

\begin{tabular}{|c|c|c|c|c|c|c|c|c|c|c|}
\hline \multicolumn{6}{|c|}{ CONCORDIA COLUMNS } & \multicolumn{2}{|c|}{$<1000 \mathrm{Ma}$} & \multicolumn{2}{|c|}{$>1000 \mathrm{Ma}$} & \multirow{3}{*}{ Conc. Age } \\
\hline \multirow[t]{2}{*}{ File } & \multirow{2}{*}{$207 / 235$} & \multirow{2}{*}{$7 / 5 \mathrm{err}$} & \multirow{2}{*}{$206 / 238$} & \multirow{2}{*}{$6 / 8 \mathrm{err}$} & \multirow{2}{*}{ Rho } & $\begin{array}{c}206 \mathrm{~Pb} / \\
238 \mathrm{U}\end{array}$ & $\begin{array}{c}206 \mathrm{~Pb} / \\
238 \mathrm{U}\end{array}$ & $\begin{array}{l}207 \mathrm{~Pb} / \\
206 \mathrm{~Pb}\end{array}$ & $\begin{array}{l}207 \mathrm{~Pb} / \\
206 \mathrm{~Pb}\end{array}$ & \\
\hline & & & & & & Age & 2sigma & Age & 2sigma & \\
\hline HH-17-40_44_1 & 11.49 & 0.4 & 0.488 & 0.014 & 0.5236 & 2557 & 61 & 2565 & 49 & 2563.75 \\
\hline HH-17-40_43_1 & 11.54 & 0.34 & 0.491 & 0.013 & 0.59159 & 2570 & 55 & 2545 & 36 & 2567.28 \\
\hline HH-17-40_46_1 & 7.82 & 0.2 & 0.4082 & 0.0094 & 0.43673 & 2212 & 40 & 2204 & 34 & 2210.26 \\
\hline HH-17-40_46_2 & 7.76 & 0.15 & 0.4063 & 0.0071 & 0.61541 & 2196 & 33 & 2213 & 19 & 2203.97 \\
\hline HH-17-40_49_1 & 4.794 & 0.1 & 0.3198 & 0.0061 & 0.51335 & 1787 & 30 & 1769 & 26 & 1784.14 \\
\hline HH-17-40_48_1 & 4.8 & 0.18 & 0.3168 & 0.0088 & 0.47186 & 1772 & 43 & 1765 & 59 & 1782.44 \\
\hline HH-17-40_40_1 & 5.965 & 0.13 & 0.3479 & 0.0066 & 0.55629 & 1922 & 31 & 2027 & 24 & 1968.77 \\
\hline HH-17-40_40_2 & 4.85 & 0.16 & 0.3204 & 0.0078 & 0.17691 & 1791 & 37 & 1767 & 61 & 1793.00 \\
\hline HH-17-40_2_1 & 5.48 & 0.13 & 0.3411 & 0.0068 & 0.4847 & 1891 & 33 & 1887 & 35 & 1896.86 \\
\hline
\end{tabular}


Vol. 58 No 04 (228) 2018

\begin{tabular}{|c|c|c|c|c|c|c|c|c|c|c|}
\hline HH-17-40_2_2 & 6.3 & 0.16 & 0.3663 & 0.0082 & 0.34815 & 2010 & 39 & 2003 & 42 & 2017.54 \\
\hline HH-17-40_46_3 & 5.438 & 0.12 & 0.3409 & 0.0059 & 0.39189 & 1890 & 28 & 1873 & 33 & 1890.89 \\
\hline HH-17-40_24_1 & 5.992 & 0.12 & 0.3589 & 0.0066 & 0.75429 & 1978 & 32 & 1957 & 18 & 1974.15 \\
\hline HH-17-40_20_1 & 6.02 & 0.22 & 0.351 & 0.013 & 0.54167 & 1934 & 59 & 2022 & 59 & 1979.48 \\
\hline HH-17-40_45_1 & 0.432 & 0.021 & 0.0581 & 0.0014 & 0.23421 & 363.9 & 8.4 & 290 & 97 & 364.16 \\
\hline HH-17-40_42_1 & 0.618 & 0.019 & 0.0801 & 0.0015 & 0.27943 & 496.5 & 9.1 & 408 & 60 & 494.18 \\
\hline HH-17-40_51_1 & 0.2812 & 0.009 & 0.03965 & 0.00075 & 0.08217 & 250.6 & 4.7 & 235 & 65 & 250.93 \\
\hline HH-17-40_38_1 & 0.421 & 0.013 & 0.0576 & 0.0013 & 0.37915 & 361.1 & 8.1 & 307 & 60 & 359.42 \\
\hline HH-17-40_34_1 & 0.2738 & 0.0091 & 0.03951 & 0.00077 & 0.11332 & 249.8 & 4.7 & 171 & 67 & 248.65 \\
\hline HH-17-40_34_2 & 0.278 & 0.012 & 0.03879 & 0.001 & 0.32161 & 245.3 & 6.4 & 272 & 93 & 246.12 \\
\hline HH-17-40_32_1 & 0.618 & 0.021 & 0.0792 & 0.0016 & 0.07999 & 491 & 9.5 & 434 & 76 & 490.43 \\
\hline HH-17-40_30_1 & 0.646 & 0.032 & 0.0802 & 0.0025 & 0.53114 & 497 & 15 & 535 & 77 & 499.19 \\
\hline HH-17-40_28_1 & 0.46 & 0.027 & 0.0592 & 0.0026 & 0.24449 & 371 & 16 & 490 & 140 & 375.94 \\
\hline HH-17-40_28_2 & 0.459 & 0.014 & 0.0603 & 0.0012 & 0.26483 & 377.5 & 7.5 & 382 & 57 & 379.34 \\
\hline HH-17-40_27_1 & 0.615 & 0.024 & 0.0784 & 0.0019 & 0.43464 & 486.7 & 11 & 442 & 74 & 486.60 \\
\hline HH-17-40_26_1 & 0.267 & 0.033 & 0.0372 & 0.0023 & 0.06368 & 236 & 14 & 240 & 270 & 236.47 \\
\hline НH-17-40_25_1 & 0.437 & 0.019 & 0.0582 & 0.0013 & 0.09565 & 364.4 & 8.2 & 359 & 99 & 365.63 \\
\hline HH-17-40_23_1 & 0.458 & 0.018 & 0.0599 & 0.0017 & 0.44422 & 374.7 & 10 & 400 & 73 & 377.59 \\
\hline HH-17-40_22_1 & 0.46 & 0.018 & 0.0607 & 0.0015 & 0.43467 & 380 & 8.9 & 366 & 68 & 380.91 \\
\hline HH-17-40_15_2 & 0.618 & 0.019 & 0.0791 & 0.0017 & 0.29184 & 490.5 & 10 & 452 & 60 & 489.91 \\
\hline HH-17-40_14_1 & 0.453 & 0.023 & 0.0611 & 0.0014 & 0.10414 & 381.9 & 8.7 & 280 & 100 & 381.75 \\
\hline HH-17-40_12_1 & 0.277 & 0.015 & 0.0383 & 0.0014 & 0.48426 & 241.9 & 8.8 & 275 & 98 & 243.56 \\
\hline HH-17-40_11_1 & 0.395 & 0.013 & 0.05402 & 0.0011 & 0.15163 & 339 & 6.9 & 293 & 66 & 338.80 \\
\hline HH-17-40_11_2 & 0.3959 & 0.011 & 0.0537 & 0.00095 & 0.22526 & 337.1 & 5.8 & 310 & 52 & 337.64 \\
\hline HH-17-40_8_1 & 0.391 & 0.017 & 0.0537 & 0.0012 & 0.188 & 336.8 & 7.3 & 255 & 84 & 336.75 \\
\hline HH-17-40_4_1 & 0.568 & 0.018 & 0.0731 & 0.0016 & 0.35274 & 454.7 & 9.3 & 449 & 59 & 455.48 \\
\hline HH-17-40_3_1 & 0.428 & 0.016 & 0.0571 & 0.0014 & 0.21038 & 357.9 & 8.6 & 337 & 80 & 359.19 \\
\hline HH-17-40_3_2 & 0.454 & 0.017 & 0.0599 & 0.0013 & 0.14293 & 374.8 & 7.7 & 360 & 76 & 376.42 \\
\hline
\end{tabular}



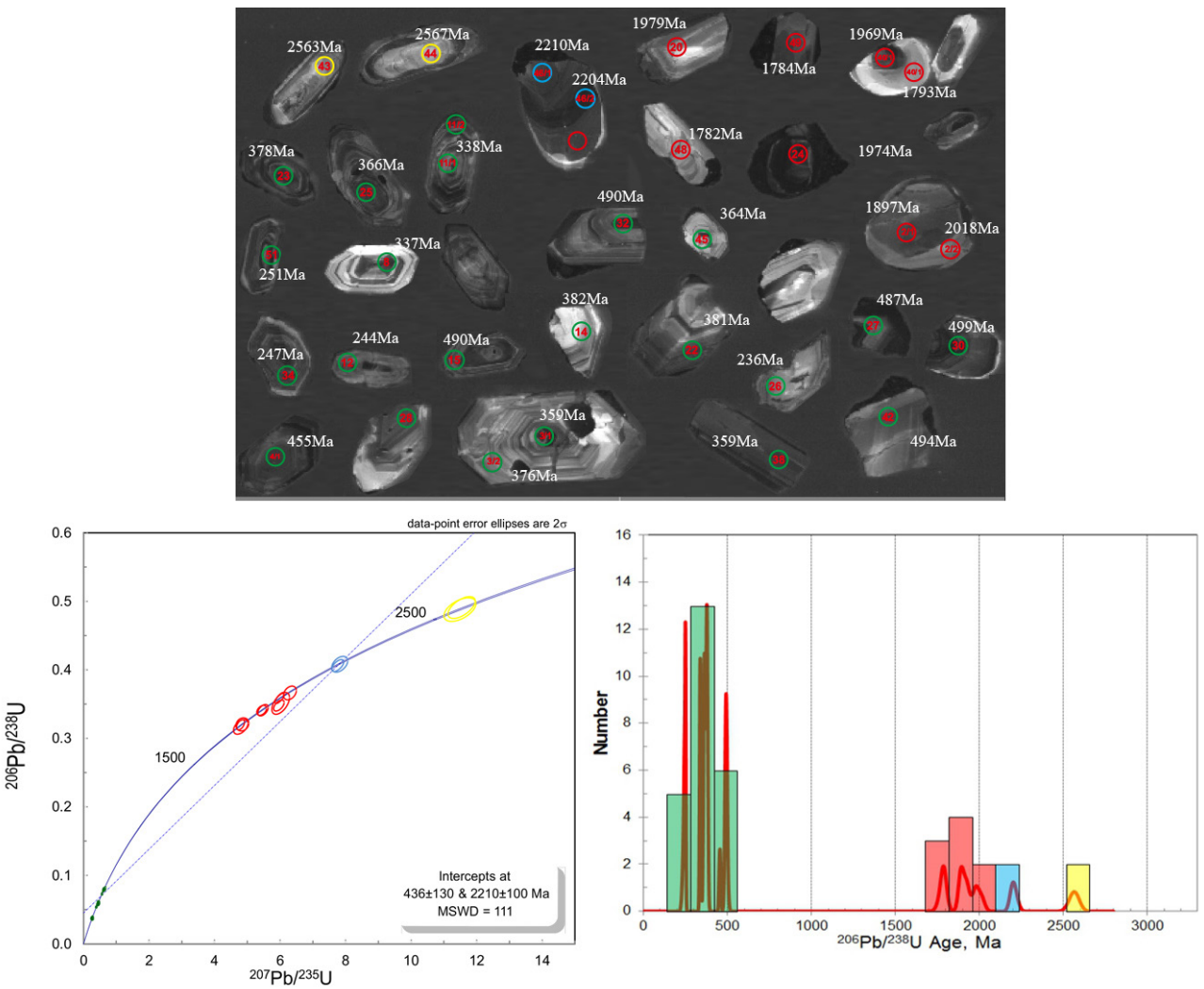

Figure 9a) Representative CL images of the detrital zircon, b) Condordia plot of detrital zircon, and,

c) Detrital zircons age histogram

\section{Depositional period of the sedimentary rocks at Shar Khutul area}

Detrital zircon (HH-17-40) from Tsetserleg formation yielded ages between $2.5 \mathrm{Ga}$ and 236 $\mathrm{Ma}$, and were grouped in four main peaks: (1) 2.5-1.7Ga (n=13), (2) 499-455Ma ( $=6),(3)$ $382-337 \mathrm{Ma}(\mathrm{n}=13)$ and (4) 250-236Ma $(\mathrm{n}=5)$. We regard that the third main peak can indicate depositional period, because the fourth peak would be related to reaction of metamorphism, derived from the Khangai intrusion complex $\left(\mathrm{P}_{2}-\mathrm{T}_{1}\right)$ or weathering.

The ancient peak is $2.5-1.7 \mathrm{Ga}$ and corresponds to the Paleoproterozoic basement in the Baidrag block $[13,5]$, the second peak (499-455Ma) corresponds to the Dzag series sediments [14], and the fourth peak (250236Ma) includes 5 zircon grains, which correspond to Middle Permian-Early Triassic Khangai intrusive complex $[42,25,26]$. The zircon grains and morphology of the fourth peak represent re-crystallization event. It is probably related to Middle Permian-Early Triassic granitoid pluton.

In the previous literatures, our newly classified Tsetserleg formation belonged to Lower-Middle Devonian Erdenetsogt formation. Based on geochronology, the Tsetserleg formation is assigned Upper Devonian-Lower Carboniferous periods. Otherwise, the Tsetserleg formation should be distinguished for lithology from the Erdenetsogt formation. In addition, according to radiolarian study, we classified that the 
Erdenetsogt formation is Upper Silurian Middle Devonian period, which is composed of basalt, quartzite, chert, minor siliceous

\section{Sorting and weathering effects}

The sedimentary sorting and recycling can be monitored by a plot of $\mathrm{Th} / \mathrm{Sc}$ versus $\mathrm{Zr} / \mathrm{Sc}$ [19]. A simple positive correlation between $\mathrm{Th} / \mathrm{Sc}$ and $\mathrm{Zr} / \mathrm{Sc}$ ratio is exhibited by first cycle sediments, whereas there is a substantial increase in $\mathrm{Zr} / \mathrm{Sc}$ with far less increase in $\mathrm{Th} /$ $\mathrm{Sc}$ in recycled sediments $[1,31]$. On the $\mathrm{Th} /$ $\mathrm{Sc}$ versus $\mathrm{Zr} / \mathrm{Sc}$ diagram, the sandstones of the Shar Khutul are shown simple positive correlation, which is consistent with that of the first cycle sediments (Figure 10).

The $\mathrm{Th} / \mathrm{U}$ ratio is also a useful parameter in determining the source characteristics of

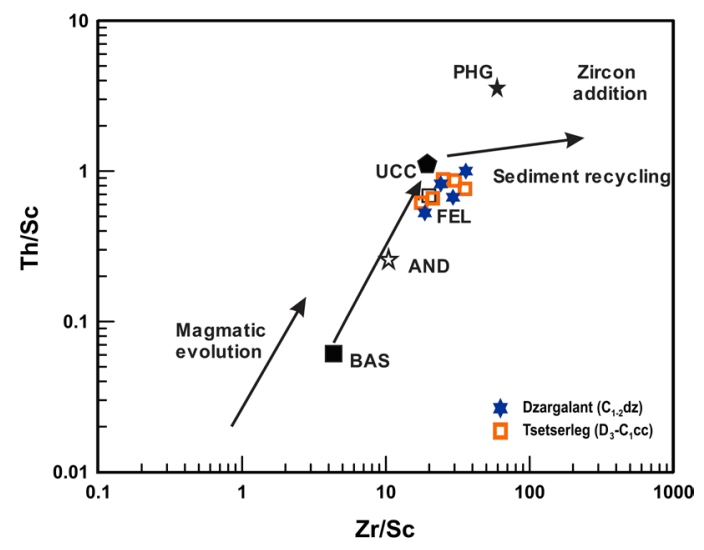

Figure 10. Plot of Th/Sc against Zr/Sc [19]

The higher chemical index of alteration (CIA), ranging between 76 and 100, indicates the intensive chemical weathering in the source areas $[22,11]$, whereas values $<50$ indicate near absence of chemical alteration and reflect cool and arid conditions or unweathered source areas [11]. Furthermore, sandstones with index of compositional variation (ICV) $>1$ are compositionally immature with the first cycle of sediments deposited in tectonically active settings and $\mathrm{ICV}<1$ are compositionally mature and were deposited in the quiescent or cratonic environment where sediment recycling was active [6]. In the present study, the CIA siltstone, mudstone, probably none of sandstone.

clastic sedimentary rocks [32]. In sedimentary rocks, $\mathrm{Th} / \mathrm{U}$ values higher than 4.0 may indicate intense weathering in source areas or sediment recycling [20]. In this study, all sandstones $\mathrm{Th} / \mathrm{U}$ ratios range between 3.60 and 3.89, $(\mathrm{av} .=3.75)$. This suggests that the sandstones were derived from unweathered rocks, which indicate they were similar to UCC $(\mathrm{Th} / \mathrm{U}=3.80)$. The $\mathrm{Th} / \mathrm{U}$ versus $\mathrm{Th}$ plot for the sandstones (Figure 11)shows typical distribution similar to the average values of the upper continental crust and follows the normal weathering trend [19].

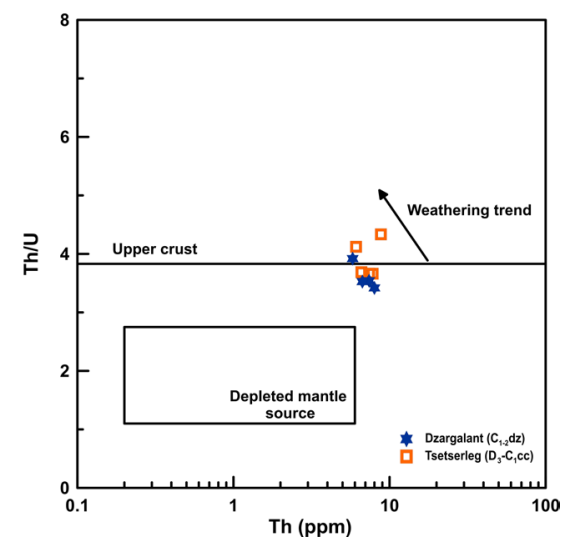

Figure 11. Plot of Th against Th/U [19]

values range between 48.71 and 56.94, with an average of 52.21, while ICV values range between 0.98 and 1.24 with an average of 1.12 (Table 1).The binary plot of CIA against ICV for studied samples shows that most of the sandstones are geochemically immature and were derived from weak weathered source rocks (Figure 13).

The CIA values of the studied sandstones are also plotted in the $\mathrm{Al}_{2} \mathrm{O}_{3}-\left(\mathrm{CaO}^{*}+\mathrm{Na}_{2} \mathrm{O}\right)$ $\mathrm{K}_{2} \mathrm{O}(\mathrm{A}-\mathrm{CN}-\mathrm{K})$ diagram, which may express much of the chemical variation resulting from weathering. Unweathered rocks cluster along the left-hand side of the plagioclase-K- 
feldspar join line in the A-CN-K system [23]. All samples of the Shar Khutul area are plotted nearly in the plagioclase-K-feldspars join line,

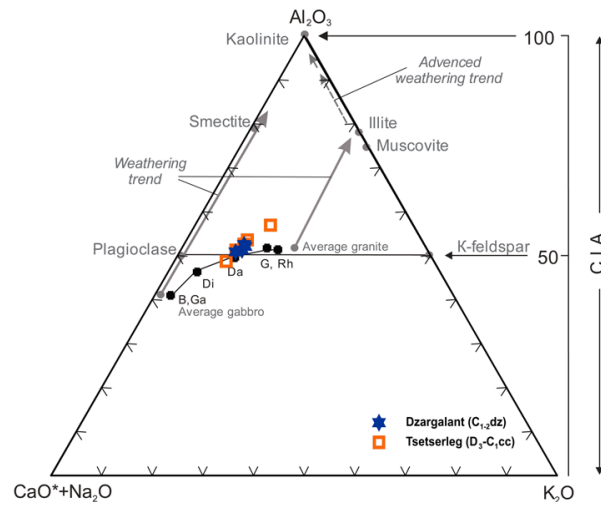

Figure 12. Al2O3-CaO $*+\mathrm{Na} 2 \mathrm{O}-\mathrm{K} 2 \mathrm{O}$ ternary diagram [23]

\section{Provenance and tectonic settings}

The $\mathrm{SiO}_{2}$ content and $\mathrm{K}_{2} \mathrm{O} / \mathrm{Na}_{2} \mathrm{O}$ ratios in sandstones appear to be particularly sensitive indicators of geotectonic setting of the source area [33]. The quartz-poor greywackes (quartz $<15 \%, \quad$ average $\mathrm{SiO}_{2}=58 \%, \quad \mathrm{~K}_{2} \mathrm{O} /$ $\left.\mathrm{Na}_{2} \mathrm{O}<1\right)$ are indicative of magmatic island arcs and quartz intermediate greywackes (quartz $=15-65 \%, \quad \mathrm{SiO}_{2}=68-74 \%, \quad \mathrm{~K}_{2} \mathrm{O} /$ $\left.\mathrm{Na}_{2} \mathrm{O}<1\right)$ are indicative of Andean-type continental margins and have approximately the same composition as the upper continental crust. Quartz-rich greywackes (quartz $>65 \%$, $\left.\mathrm{SiO}_{2}=89 \%, \mathrm{~K}_{2} \mathrm{O} / \mathrm{Na}_{2} \mathrm{O}>1\right)$ are characteristic of Atlantic-type continental margins and are similar in composition to the sand fraction of the continental platform cover [35]. The sandstones of study area are quartz intermediate greywackes $\left(\mathrm{SiO}_{2}=67-70 \%, \mathrm{~K}_{2} \mathrm{O} /\right.$ which is perhaps because these samples were derived from felsic-intermediate igneous and unweathered source rocks (Figure 13).

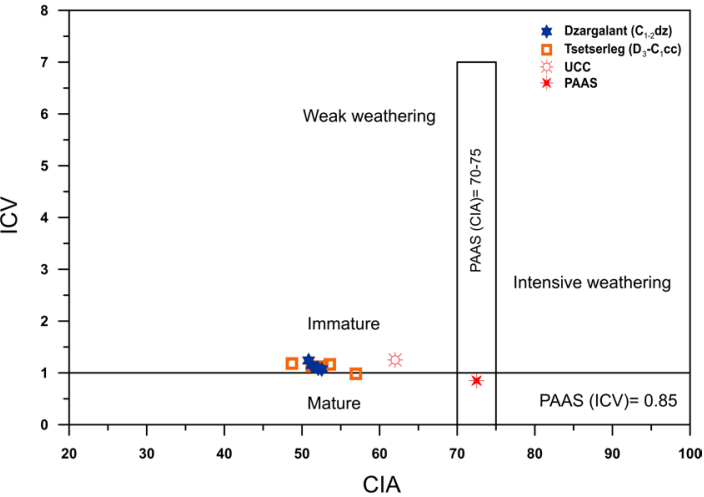

Figure 13. Binary diagram of CIA against ICV[6]

$\left.\mathrm{Na}_{2} \mathrm{O}<1\right)$ and it is similar in composition with upper continental crust.

Therefore, $\mathrm{K}_{2} \mathrm{O} / \mathrm{Na}_{2} \mathrm{O}$ ratio and $\mathrm{SiO}_{2}$ content discriminate between passive-margin and active-margin sandstones [17]. They suggest that passive-margin sandstones have $\mathrm{K} \mathrm{O}_{2} / \mathrm{Na}_{2} \mathrm{O}$ ratios greater than 1 and activemargin sandstones have ratios less than 1 . Also passive-margin sandstones are enriched in $\mathrm{SiO}_{2}$ compared to active margin sandstones.

$\mathrm{K}_{2} \mathrm{O} / \mathrm{Na}_{2} \mathrm{O}$ versus $\mathrm{SiO}_{2}$ diagram shows the studied sandstones may be classified as active continental margin provenance (Figure 14), furthermore the sandstones plotted in the field of active continental margin in $\mathrm{La} / \mathrm{Sc}-\mathrm{Ti} / \mathrm{Zr}$ diagram [3] which describes the geotectonic environments (Figure 15). 


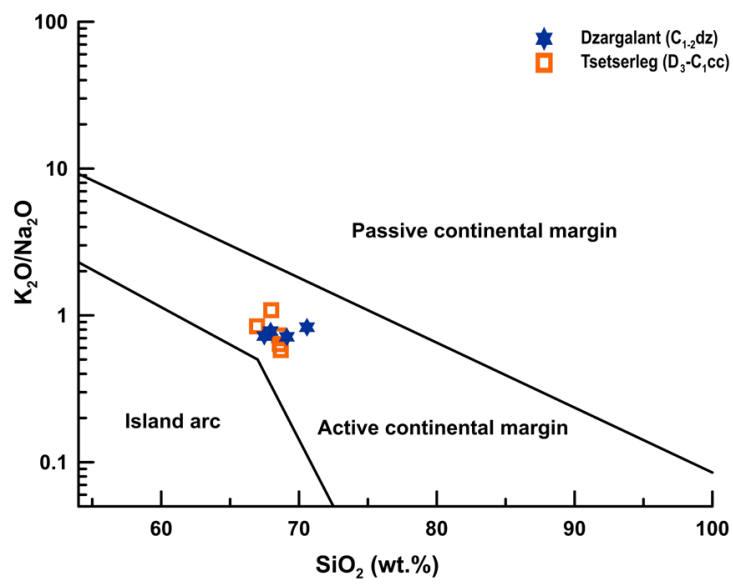

Figure 14. Tectonic discrimination diagram of Roser and Korsch [32]

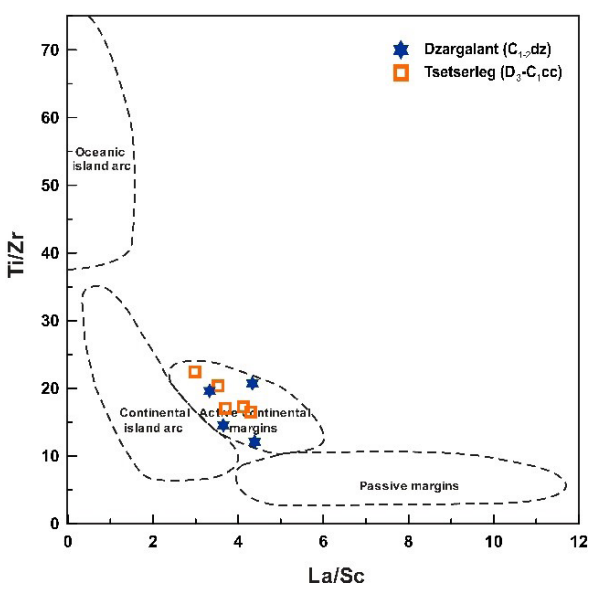

Figure 15. Ti/Zr versus La/Sc plot of the sandstones for tectonic setting discrimination [4]
Acknowledgement: This study was financially supported by "Geodynamic settings and structural criteria of gold mineralization of the Khangai-Khentey orogenic system in the Mongol-Okhotsk foldbelt" Grant (Contract No. SSA-008/2016) from the Mongolian Foundation for Science and Technology.

\section{CONCLUSIONS}

Based on petrography, geochemistry and zircon $\mathrm{U}-\mathrm{Pb}$ age dating results of the Paleozoic sedimentary rocks from the Shar Khutul area, we can draw the following conclusions.

- Framework petrography, major oxide and trace elements indicate that these sandstones were derived from felsic igneous rocks, formed probably with active continental margin provenance.

- $\quad$ The CIA values range from 48.71 to 56.94 with an average of 52.21, ICV values range from 0.98 to 1.24 with an average of 1.12. These studied samples show that most of the sandstones are geochemically immature and were derived from weak weathered source rocks.

- The sample yielded ages between $2.5 \mathrm{Ga}$ and $236 \mathrm{Ma}$, and the detrital zircons are grouped in four main peaks: (1) $1.7-2.5 \mathrm{Ga}$ $(\mathrm{n}=13)$, (2) 455 - 499Ma ( $=6)$, (3) 337$382 \mathrm{Ma}(\mathrm{n}=13)$, and, (4) $236-250 \mathrm{Ma}(\mathrm{n}=$ 5 ). We regard the third main peak, which would indicate depositional period, but the fourth peak would be related to reaction of metamorphism derived from the Khangai intrusion complex (P2-T1).

\section{REFERENCES}

[1] Aseidu, D.K., Suzuki, S., Nogami, K., and Shibata, T., 2000, Geochemistry of Lower Cretaceous sediments, Inner zone of southwest Japan: Constraints on provenance and tectonic environment, Geochemical J, 34, pp. 155-173.

[2] Batdelger, D., Khishigsuren, S., Orolmaa, D., Myagmarjav, Ganbayar, B., 2015, Petrography, geochemistry, and geodynamic environment of the Lower-Middle Devonian sandstone in the Erdenetsogt Formation, Journal of Prospector, 54, pp. 63 76 (In Mongolian). 
[3] Bhatia, M.R., 1983, Plate tectonics and geochemical composition of sandstones, Journal of Geology, 91, pp. 611-627.

[4] Bhatia, M.R., and Crook, K.A.W., 1986, Trace element characteristics of greywacke and tectonic setting discrimination of sedimentary basins, Contributions to Mineralogy and Petrology, 92, pp. 181-193.

[5] Bibikova, E. V., Baikova, V. S., Gorohovskii, B. M., Gracheva, T. V., Kirnozova, T. I., Kozakov, I. K., Kotov, A.B., et al. 1990, Early Proterozoic boundary in the Baidrag Block in central Mongolia, Izv. Akad. Nauk SSSR Ser. Geol. 7:57-62 (in Russian).

[6] Cox, R., Lowe, D.R., and Cullers, R.L., 1995. The influence of sediment recycling and basement composition of evolution of mudrock chemistry in the southwestern United States, Geochim Cosmocim Acta, 59, pp. 2919-2940.

[7] Cullers, R. L., and Graf, J., 1983, Rare earth elements in igneous rocks of the continental crust: intermediate and silicic rocks, ore petrogenesis; In: Rare-Earth Geochemistry (ed.) Henderson P., Elsevier, Amsterdam, pp. 275-312.

[8] Cullers, R. L., 1994, The controls on the major and trace element variation of shales, siltstones, and sandstones of Pennsylvanian-Permain age from uplifted continental blocks in Colorado to platform sediment in Kansas, USA, Geochimica et Cosmochimica Acta, 58, pp. 4955-4972.

[9] Cullers, R. L., 2000, The geochemistry of shales, siltstones and sandstones of Pennsylvanian-Permian age, Colorado, USA: implications for provenance and metamorphic studies, Lithos 53(3), pp. 181-203.

[10] Erdenechimeg, D., Oyunchimeg T., Tumurchudur Ch., Dagva-Ochir L., Jitka Mikova, Otgonbaatar D., Enkhdalai B., Delgerzaya, P., 2018, Geochemistry and provenance of sedimentary rocks in the Shar Khutul area, Central Mongolia, Journal of Geological Issues, GIMAR, 494(16), p.170.

[11] Fedo, C. M., Nesbitt, H .M., Young, G. M., 1995, Unraveling the effects of potassium metasomatism in sedimentary rocks and paleosols, with implications for paleoweathering conditions and provenance. Geology, 23, pp. 921-924.

[12] Gurtsoo, S., Tileyu, D., Rinchindorj, Sh., Baljinnyam, D., Narangerel, S., Batjargal, M. (1990), Report on 1:200000 exploration survey carried out in Bayankhongor, Ovorkhangai and Arkhangai aimags by Ikhtamir group in 1986-1990, № 4417 (in Mongolian).

[13] Kovalenko, V. I., Yarmolyuk, V. V., Tomurtogoo, O., Antipin, V. S., Kovach, V. P., Kotov, A. B., Kudryashova, E. B., Sal'nikova, E. B., and Zagornaya, N. Y., 2005, Geodynamics and crust-forming processes in the early Caledonides of the Bayanhongor Zone, central Mongolia, Geotectonics 39:298-31.

[14] Kröner, A., Demoux, A., Zack, T., Rojas-Agramonte, Y., Jian, P., Tomorkhuu, D., Barth, M., 2011, Zircon ages for a felsic volcanic rock and arc-related early Palaeozoic sediments on the margin of the Baydrag microcontinent, central Asian orogenic belt, Mongolia, Journal of Asian Earth Sciences 42, pp. 1008-1017.

[15] Lhundev, Sh., Tomorchudur, Ch., Purevdorj, Z., Baadai, D., Unurjav, L. and Ganbold, Yu., (1994), Geology and mineral resources of the Uyanga area 1:50000. Geological mapping and prospecting report 4756, p. 619, Ulaanbaatar, Mongolia. (in Mongolian).

[16] Luo, Z., Zhang, Z., Li, K. ,Li, J., Tang, W., and Xu, B., 2015, Petrography, geochemistry, and $\mathrm{U}-\mathrm{Pb}$ detrital zircon dating of early Permian sedimentary rocks from the North Flank of the North China Craton: Implications for the late Palaeozoic tectonic evolution of the eastern Central Asian Orogenic Belt, International Geology review, 58, pp. 787- 
806.

[17] Maynard, J. B., Valloni, R., and Yu, H. S., 1982, Composition of the modern deep-sea sands from arc related basins; In: Trench-forearc geology sedimentation and tectonics on modern and ancient active plate margins, (ed.) Leggett, J. K., Geol. Soc. London Publ. 10, pp. 551-561.

[18] McLennan, S. M., and Taylor, S. R., 1991, Sedimentary rocks and crustal evolution: Tectonic setting and secular trends. J. Geol., 99, pp. 1-21.

[19] McLennan, S. M., Hemming, S., McDaniel, D. K., Hanson, G. N., 1993, Ceochemical approaches to sedimentation, provenance and tectonics. In: Johnsson, M.J., Basu, A. (Eds.), Processes Controlling the Composition of Clastic Sediments, Geological Society of America Special Paper, 284, pp. 21-40.

[20] McLennan, S. M., Hemming, S. R., Taylor, S. R., and Eriksson, K. A., 1995, Early Proterozoic crustal evolution: Geochemical and $\mathrm{Nd}-\mathrm{Pb}$ isotopic evidence from metasedimentary rocks, southern North America. Geochim. Cosmochim. Acta, 59, pp. $1153-1177$.

[21] McLennan, S. M., 2003, The roles of provenance and sedimentary processes in the geochemistry of sedimentary rocks, In: Lentz, D.R., (Ed.), Geochemistry of Sediments and Sedimentary Rocks: Evolutionary Considerations to Mineral Deposit-Forming Environments. Geological of Association of Canada, Newfoundland, pp. 7-38.

[22] Nesbitt, H. W., Young, G. M., 1982, Early Proterzoic climates and plate motions inferred from major element chemistry of lutites, Nature, 299, pp. 715-717.

[23] Nesbitt, H. W., Young, G. M, 1984, Prediction of some weathering trends of plutonic and vulcanic rocks based on thermodynamic and kinetic consideration, Geochimica et Cosmochimica Acta, 48, pp. 1523-1534.

[24] Orolmaa, D., Erdenesaikhan, G., 2008, Geology and geochemical characteristic of Devonian basalt from the Khangai region, Treatise of IGMR, MAS, 18, pp. 50-60 (In Mongolian)

[25] Orolmaa, D., Erdenesaikhan, G., Borisenko, A. S., Fedoseev, G. S., Babich, V. V., Zhmodik, S. M., 2008, Permian-Triassic granitoid magmatism and metallogeny of the Khangayn (central Mongolia), Russ. Geol. Geophys, 49, pp. 534-544.

[26] Orolmaa, D., Erdenesaikhan, G., 2009, Geochemistry and isotopic age of the Khangai granitoid, Mongolian geoscientist, 34, pp. 100-105 (In Mongolian).

[27] Oyunchimeg, T., Enkhdalai, B., Otgonbaatar, B., Jitka Mikova, Erdenechimeg, D., Sharav, D., Dagva-Ochir, L., Battushig, A., 2018, Geochemistry of the Paleozoic sedimentary rocks in Tsoroidog area, Tsetserleg terrane, Treatise of IPG, MAS, 24, pp. 6-30 (In Mongolian).

[28] Pettijohn, F. J., Potter, P. E., Siever, R., 1987. Sand and Sandstone: New York, SpringerVerlag,. New York, Xvi, p. 618.

[29] Purevjav, N., and Roser, B. P., 2012, Geochemistry of Devonian-Carboniferous clastic sediments of the Tsetserleg terrane, Khangai Basin, Central Mongolia: Provenance, source weathering, and tectonic setting, Island Arc, 21, pp. 270-287.

[30] Purevjav, N., and Roser, B. P., 2013, Geochemistry of Silurian-Carboniferous sedimentary rocks of the Ulaanbaatar terrane, Khangai-Khentii belt, Central Mongolia: Provenance, paleoweathering, tectonic setting, and relationship with the neighbouring Tsetserleg terrane, Chemie der Erde, 73, pp. 1-13.

[31] Rahman, M. J., and Suzuki, S., 2007, Geochemistry of sandstones from the Miocene Surma Group, Bengal basin, Bangladesh: Implications for provenance, tectonic setting 
and weathering, Geochem.J, 41, pp. 415-428.

[32] Roddaz, M., Viers, J., Brusset, S., Baby, B., Boucayrand, C., and Herail, G., 2006. Controls on weathering and provenance in the Amazonian foreland basin: Insights from major and trace element geochemistry of Neogene Amazonian sediments, Chem. Geol, 226, pp. 31-65.

[33] Roser, B. P., Kosch, R.J., 1986, Determination of tectonic setting of sandstone and mudstone suites using $\mathrm{SiO} 2$ and $\mathrm{K} 2 \mathrm{O} / \mathrm{Na} 2 \mathrm{O}$ ratio, Journal of Geology, 94, pp. 635650.

[34] Roser, B. P., Kosch, R. J., 1988, Provenance signatures of sandstone-mudstone suites determined using discriminant function analysis of major element data, Chemical Geology, 67, pp. 119-139.

[35] Schwab, F. L., 1975, Framework mineralogy and chemical composition of continental margin-type sandstone: Geology, v. 3, no. 9, pp. 487-490.

[36] Sun, S.S., and McDonough, W.F., 1989. Chemical and isotopic systematics of oceanic basalts: implications for mantle composition and processes. In: Saunders A.D. and Norry M.J. (eds) Magmatism in ocean basins, Geol. Soc. London, Spec. Pub. 42, pp. 313-345.

[37] Taylor, S. R., McLennan, S. M., 1985, The Continental Crust: its Composition and Evolusion, Blackwell, Oxford, p. 312.

[38] Tomorchudur, Ch., Ganbat, L., Noosoy, Z., Enkhsaikhan, Ts., (1990), Report on 1:200000 exploration survey carried out in Bayankhongor, Ovorkhangai and Arkhangai aimags by Galuut group in 1986-1990, № 4415 (in Mongolian).

[39] Tomortogoo, O., 2012. Tectonic subdivision orogenic belt of Mongolia. Treatise of IGMR, MAS, 21, pp. 5-25 (in Mongolian).

[40] Wang, W., Zhou, M. F., 2012, Sedimentary records of the Yangtze Block (South China) and their correlation with equivalent Neoproterozoic sequences on adjacent continents, Sedimentary Geology, pp. 265-266, and 126-142.

[41] Wang, W., Zhou, M. F., Yan, D. P., Li, J. W., 2012, Depositional age, provenance, and tectonic setting of the Neoproterozoic Sibao Group, southeastern Yangtze Block, South China, Precambrian Research, pp. 192-195, 107-124.

[42] Yarmolyuk, V. V., Kovalenko, V. I., Kozakov, I. K., Sal'nikova, E. B., Bibikova, E. V., Kovach, V. P., Kozlovsky, A. M., et al. 2008b, The age of the Khangai batholith and the problem of batholith Formation in central Asia. Dokl. Earth Sci. 423:1123-1128. 